\title{
Spontaneous Network Coupling Enables Efficient Task Performance without Local Task-Induced Activations
}

\author{
${ }^{\circledR}$ Leslie Allaman, ${ }^{1}$ Anaïs Mottaz, ${ }^{1}$ Andreas Kleinschmidt, ${ }^{2}$ and ${ }^{\circledR}$ Adrian G. Guggisberg ${ }^{1}$ \\ ${ }^{1}$ Division of Neurorehabilitation, Department of Clinical Neurosciences, University Hospital of Geneva, Genève, GE 1211, Switzerland, and \\ ${ }^{2}$ Division of Neurology, Department of Clinical Neurosciences, University Hospital of Geneva, Genève, GE 1205, Switzerland
}

Neurobehavioral studies in humans have long concentrated on changes in local activity levels during repetitive executions of a task. Spontaneous neural coupling within extended networks has latterly been found to also influence performance. Here, we intend to uncover the underlying mechanisms, the relative importance, and the interaction between spontaneous coupling and task-induced activations. To do so, we recorded two groups of healthy participants (male and female) during rest and while they performed either a visual perception or a motor sequence task. We demonstrate that, for both tasks, stronger activations during the task as well as greater network coupling through spontaneous $\alpha$ rhythms at rest predict performance. However, high performers present an absence of classical task-induced activations and, instead, stronger spontaneous network coupling. Activations were thus a compensation mechanism needed only in subjects with lower spontaneous network interactions. This challenges classical models of neural processing and calls for new strategies in attempts to train and enhance performance.

Key words: event-related desynchronization; motor planning; neural coupling; visual perception

Significance Statement

Our findings challenge the widely accepted notion that task-induced activations are of paramount importance for behavior. This will have an important impact on interpretations of human neurobehavioral research. They further link the widely used techniques of quantifying network communication in the brain with classical neuroscience methods and demonstrate possible ways of how network communication influences human behavior. Traditional training methods attempt to enhance neural activations through task repetitions. Our findings suggest a more efficient neural target for learning: enhancing spontaneous neural interactions. This will be of major interest for a large variety of scientific fields with very broad applications in schools, work, and others.

\section{Introduction}

The understanding of how the brain resolves basic behavioral problems, such as sensory perception or motor planning, has been one of the central questions of science. Over the last decades, neuroscience has accumulated evidence that the successful performance of behavioral tasks is associated with patterns of neural activations at specialized brain areas during the task. Activations can be observed with brain imaging as changes in a neural signal during the task compared with a baseline signal, which is typically measured in the absence of a specific task or stimulation (Fig. 1A). When investigating field potentials

Received May 15, 2020; revised Aug. 18, 2020; accepted Sep. 12, 2020.

Author contributions: L.A. and A.M. performed research; L.A., A.M., and A.G.G. analyzed data; L.A. and A.G.G. wrote the paper; A.K. edited the paper; A.G.G. designed research; A.G.G. contributed unpublished reagents/analytic tools.

This work was supported by Swiss National Science Foundation Grant 320030_ 169275 to A.G.G.

The authors declare no competing financial interests.

Correspondence should be addressed to Adrian G. Guggisberg at aguggis@gmail.com.

https://doi.org/10.1523/JNEUROSCI.1166-20.2020

Copyright $\odot 2020$ the authors induced by large neural assemblies, task-induced activations manifest as an increase of fast oscillations in the so-called high- $\gamma$ frequency range $(>60 \mathrm{~Hz})$ and a reduction of slower rhythms (Crone et al., 2006). Since the first recordings of neural activity in humans, it is known that neural assemblies spontaneously produce prominent oscillations of an electromagnetic potential, in particular at a frequency of $\sim 8$-12 cycles per second, even when the recorded subjects are at rest. These spontaneous oscillations, named $\alpha$, are suppressed when subjects engage in a task (Pfurtscheller et al., 1996). Importantly, $\alpha$ suppression has been shown to improve stimulus perception (Ergenoglu et al., 2004; Babiloni et al., 2006; Hanslmayr et al., 2007), speed of detection (Thut et al., 2006), discrimination (van Dijk et al., 2008), as well as lateralized visuospatial attention (Thut et al., 2006; Romei et al., 2010). Therefore, the posterior $\alpha$ rhythm has for long been viewed as reflecting processes of attentional disengagement and being intrinsically inhibitory (Pfurtscheller et al., 1996; Klimesch et al., 2007; Jensen and Mazaheri, 2010). The suppression of $\alpha$ and $\beta$ amplitudes, often referred to as event-related desynchronization 
(ERD), is a widely used index of brain activation (Pfurtscheller and Lopes da Silva, 1999).

Nevertheless, more recent research showed that there is more to task-free baseline states and $\alpha$ oscillations than inhibition. A large body of research has demonstrated that spontaneous brain activity is organized into coherent networks (Greicius et al., 2009). Neural coupling (i.e., functional connectivity [FC]) between brain areas preceding a task facilitates perception and behavioral performance (Weisz et al., 2014; Sadaghiani et al., 2015; Strauß et al., 2015; Iemi et al., 2019), thus suggesting that network coupling plays an active role (Fig. 1B). This also applies to longer, spontaneous, task-independent neural coupling, in particular in $\alpha$ frequencies, which is positively correlated with performance (Hipp et al., 2011; Guggisberg et al., 2015). For instance, the more spontaneous $\alpha$ activity in Broca's area is coherent with the rest of the brain, the better subjects are able to produce words (Guggisberg et al., 2015). Phase coupling of the $\alpha$ rhythm in visual areas can also predict the detection of a transient visual target (Hanslmayr et al., 2005). Patients with brain pathologies, such as stroke and Alzheimer's disease, have reduced $\alpha$ band coupling, and the degree of this reduction correlates linearly with the severity of the behavioral impairment in a variety of functions, such as language, motor function, or spatial attention (Dubovik et al., 2012, 2013).

In sum, not only neural activations during tasks, but also spontaneous network interactions are associated with successful task performance. However, their respective role and the interactions between them (Fig. 1C) are unclear. In the present study, we thus compared the role of resting-state neural coupling (FC) and taskinduced neural activations on behavior, within paradigms of visual perception and motor planning. We hypothesized that both neural patterns influence behavior. Our findings are in line with these hypotheses but go even further. Neural $\alpha$ band coupling was found to be a more efficient neural mechanism underlying perception and motor planning than task-related activations, which were a compensation mechanism necessary only in subjects with low neural coupling. This challenges the traditional notion of a primary importance of task-induced activations.

\section{Materials and Methods \\ Participants}

Twenty healthy subjects were analyzed for Experiment 1 (12 women; $27.9 \pm 4.9$ years old) and 20 for Experiment 2 (13 women; $28.7 \pm$ 5.6 years old). Two additional subjects of Experiment 1 were excluded from analysis because of poor quality of the EEG recording. All had normal or corrected-to-normal vision and no history of neurologic or psychiatric disorders and were paid for their participation. For subjects participating in Experiment 2, we inquired whether they were piano players and how many years they practiced by means of a questionnaire.

All procedures were approved by the ethical committee of the Canton of Geneva and performed according to the Declaration of Helsinki. All participants gave written informed consent after receiving explanation on the nature the experiments.

Experimental paradigm and stimulus presentation

Subjects were seated $54 \mathrm{~cm}$ away from a 23.5 inch Eizo FG2421 LCD monitor with a refresh rate of $120 \mathrm{~Hz}$.

Experiment 1. Stimulus presentation was implemented in MATLAB (The MathWorks) using the Psychophysics Toolbox extensions
(Brainard, 1997). The subjects performed a perithreshold visual detection task interspersed with resting periods (Fig. 2). This enabled us to study the two central processing patterns of interest, classical local activations and spontaneous network interaction states, within a single paradigm. Participants were cued to close their eyes by an instruction screen. The resting period lasted between 15 and $20 \mathrm{~s}$. An auditory cue signaled the end of the resting period and the imminent start of a series of trials. Participants opened their eyes and kept fixation on a central cross $\left(0.5^{\circ}\right.$ visual angle) to avoid eye movement throughout the time of each trial. Two light gray squares $\left(3 \times 3^{\circ}\right)$ were displayed on a darker gray background and served as position markers for the location of target presentation. They were centered at $8^{\circ}$ vertical and $24^{\circ}$ horizontal eccentricity from the fixation cross. After a delay of $1500 \mathrm{~ms}$, targets (circular patches of $0.5^{\circ}$ visual angle with a Gaussian envelope) were displayed for $42 \mathrm{~ms}$ (5 time frames) at perithreshold contrast in the center of the position markers, either on the left, on the right, or bilaterally. For each subject, the contrasts were chosen such that about half of presentations were consciously perceived in each condition while the others were missed (at the same external stimulation parameters, see Procedure). Each block comprised two occurrences of each experimental condition (left, right, and bilateral) and one catch trial in which no target was displayed. The order of presentation was randomized. After another $1500 \mathrm{~ms}$, a response screen was displayed and participants had to answer, for each side, whether they had seen a target using four different response buttons (two for each side). There was no time limit for response, which could be made simultaneously for both sides or in any sequential order. They were instructed to avoid guessing and only give a positive answer when they were confident that a target had been displayed. The next trial began as soon as an answer had been collected for each side.

Experiment 2. The sequential finger tapping task was designed using E-Prime 2.0 software (Psychology Software Tools). Participants were instructed to repeat a given 5-item sequence with their left hand (little finger to index) on four horizontally arranged buttons numbered left to right on a Chronos box (Psychology Software Tools; https://pstnet.com/ products/chronos/). The same sequence was trained throughout the whole experiment (1-4-2-3-1). It was continuously presented to participants while they had to perform it. During test blocks obtained for assessment of performance, they had to repeat the sequence as fast and accurately as possible during $30 \mathrm{~s}$. No feedback was given. A further block with EEG recording was obtained to reconstruct neural activations during the task. Each trial of the EEG block was composed of two consecutive sequences with a delay of $1.5 \mathrm{~s}$ between trials (end to beginning), cued with the apparition of the sequence on the screen, for a total of 150 trials. Feedback was given with a star appearing under each item correctly pressed. In case of error, participants had to repeat the item until correct.

\section{Procedure}

Experiment 1. Subjects were tested over two consecutive afternoons. On day 1 , titration runs were obtained to determine individual perithreshold contrasts for each subject and condition. The titration session comprised two runs. The first run consisted in a 1-up 1-down interlaced triple staircase procedure with a decreasing step size and provided a rough estimate of the threshold contrast for each condition. The second 


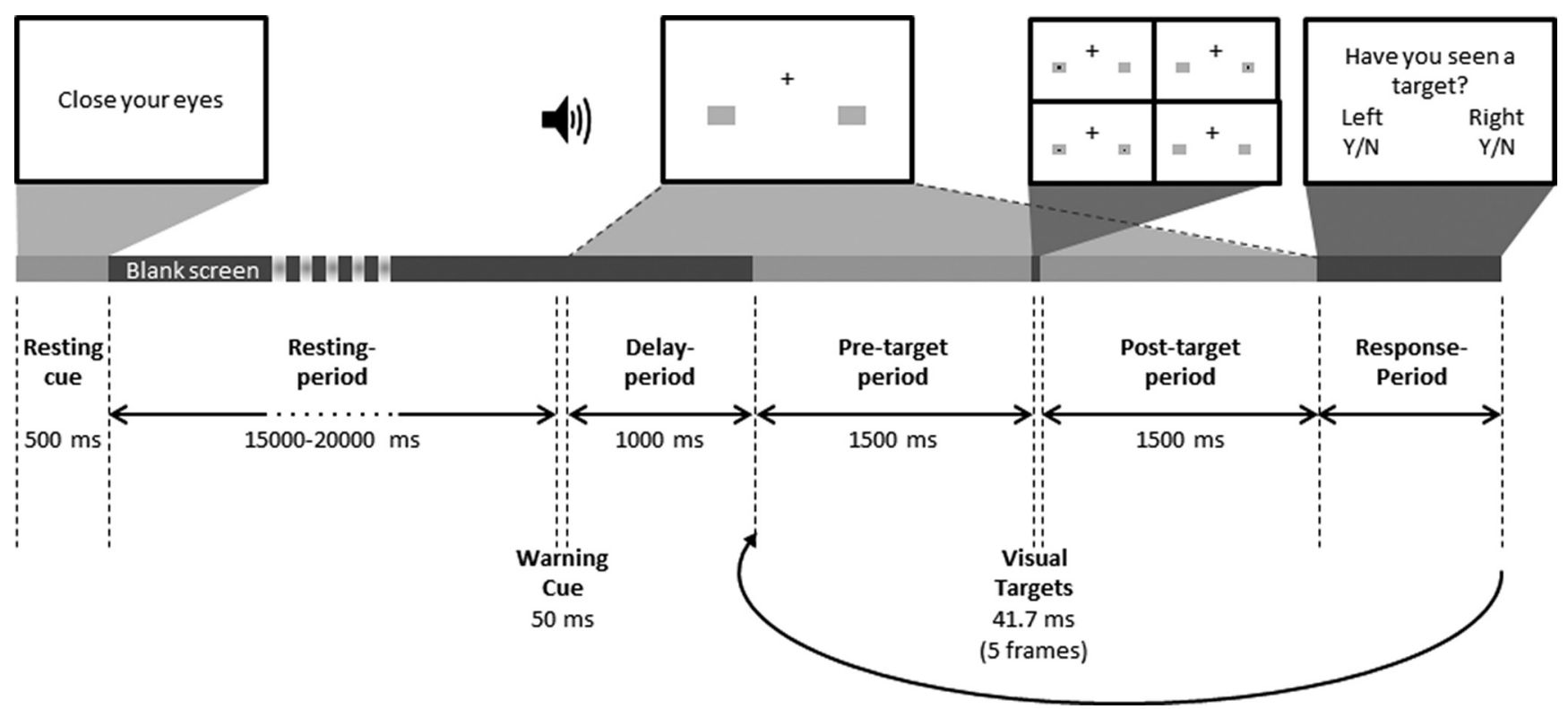

Figure 2. Experimental paradigm and time course of a single block. The task consisted of blocks starting with a resting period followed by seven trials of a target detection task (for details, see Materials and Methods). For visualization purposes, white represents the background, and dark gray represents the background of the task.

run aimed to refine the thresholds and was based on the method of constant stimuli: 6 levels of contrast were chosen for each condition based on the point of convergence of the staircase and presented 16 times each in a random order, enabling us to estimate the psychometric function for each subject and condition (Treutwein, 1995) and thus to choose contrast values with a $50 \%$ probability of detection. For bilateral trials, this meant values resulting in a $50 \%$ probability of both targets being simultaneously detected. Awareness of only one (either left or right) or none of the targets in a bilateral trial was considered an incomplete/ incorrect detection. In both runs, the three experimental conditions (left/right/bilateral) and catch trials (no target) were presented in a random order.

The EEG data were recorded during day 2. A first (shorter) run was performed to check that the subjects detected $\sim 50 \%$ of the targets. It also permitted to familiarize them with the resting part of the task. For 15 subjects, the level of contrast had to be adjusted (maximum $0.3 \%$ increase/decrease) for a least one condition. Participants then completed 120 blocks of the task, divided in 6 sessions of 20 blocks.

Experiment 2. The experiment consisted of one session. Participants completed test blocks before and after the EEG block. Participants could take a short break every 50 trials. Eyes-closed resting-state EEG was recorded during a $10 \mathrm{~min}$ before the first test block, task-induced EEG during the EEG block.

\section{Behavioral analyses}

Experiment 1. To assess compliance with instructions - participants were asked to adopt conservative criteria - we examined false alarm rates and further computed Grier's B", a nonparametric measure of response bias that ranges from -1 (maximum bias toward yes answers) to +1 (maximum bias toward no answers) (Grier, 1971; Stanislaw and Todorov, 1999). One-sample $t$ tests were used to check whether the chosen contrast values elicited a performance of $\sim 50 \%$ accuracy in our three conditions. We compared the threshold contrast values (after adjustment on recording day) between conditions using a Friedman test, given the non-normal distribution of this variable in all conditions (ShapiroFrancia tests, all $p<0.03$ ). In order to obtain an estimate of visual performance comparable across subjects, we computed the mean detection rate across conditions for contrasts that were presented to all subjects during the second titration run. Two consecutive contrast values were thus used for estimation of visual perception performance, based on 96 titration trials (16 trials for each contrast and condition). Better visual performance results in a higher visual perception percentage.
A leftward visuospatial attention bias is commonly found in young healthy subjects (Thut et al., 2006; Learmonth et al., 2015). To assess it in our subjects, we calculated the difference between the number of targets detected on the left versus on the right for bilateral trials administered on day 2. A greater bias score is found in subjects who detected the left target more often than the right.

Experiment 2. Performance was assessed as the mean number of correctly completed sequences per minute in all test blocks.

\section{EEG acquisition}

EEG data were sampled at $1024 \mathrm{~Hz}$ using a 128-channel BioSemi ActiveTwo EEG-system (BioSemi B.V.). Data were re-referenced against the $\mathrm{Cz}$ electrode. Artifacts, such as eye movements, blinks, power line, and electrode artifacts, were removed by visual inspection in Experiment 1 and for resting data of Experiment 2, and using independent components analysis (FastICA algorithm) (Hyvarinen, 1999) for task-induced epochs in Experiment 2. The artifact-free resting-state data were segmented into nonoverlapping 1s epochs.

\section{Source imaging}

All analyses were performed in MATLAB (The MathWorks), using the toolbox NUTMEG (Dalal et al., 2011) and its Functional Mapping toolbox (Guggisberg et al., 2011). Lead-potential was computed using a boundary element head model, with the Helsinki BEM library (Stenroos et al., 2007) and the NUTEEG plugin of NUTMEG (Guggisberg et al., 2011). The head model was based on the individual T1 MRI of each participant, and solution points were defined in the gray matter with 10 $\mathrm{mm}$ grid spacing. EEG data were bandpass-filtered in the respective frequency bands, Hanning windowed, Fourier transformed, and projected to gray matter voxels using an adaptive filter (scalar minimum variance beamformer) (Sekihara et al., 2004). Regions of interest (ROIs) were defined anatomically. We chose the dorsal V3 region (Eickhoff et al., 2005; Kujovic et al., 2013) for Experiment 1 since this region specifically responds to stimulation of the lower quadrant and V3 neurons have been shown to have very low-contrast thresholds (Gegenfurtner et al., 1997; Larsson and Heeger, 2006). Moreover, EEG coverage is better for dorsal parts of the visual cortex.

Concerning visuospatial attention, top-down control is thought to be mediated by right parietal areas (Gillebert et al., 2011). Accordingly, we defined the right intraparietal sulcus (see Fig. $9 A$ ) as ROI for the analyses pertaining to visuospatial bias (Eickhoff et al., 2005), using areas hIP1, hIP2 (Choi et al., 2006), and hIP3 (Scheperjans et al., 2008). 

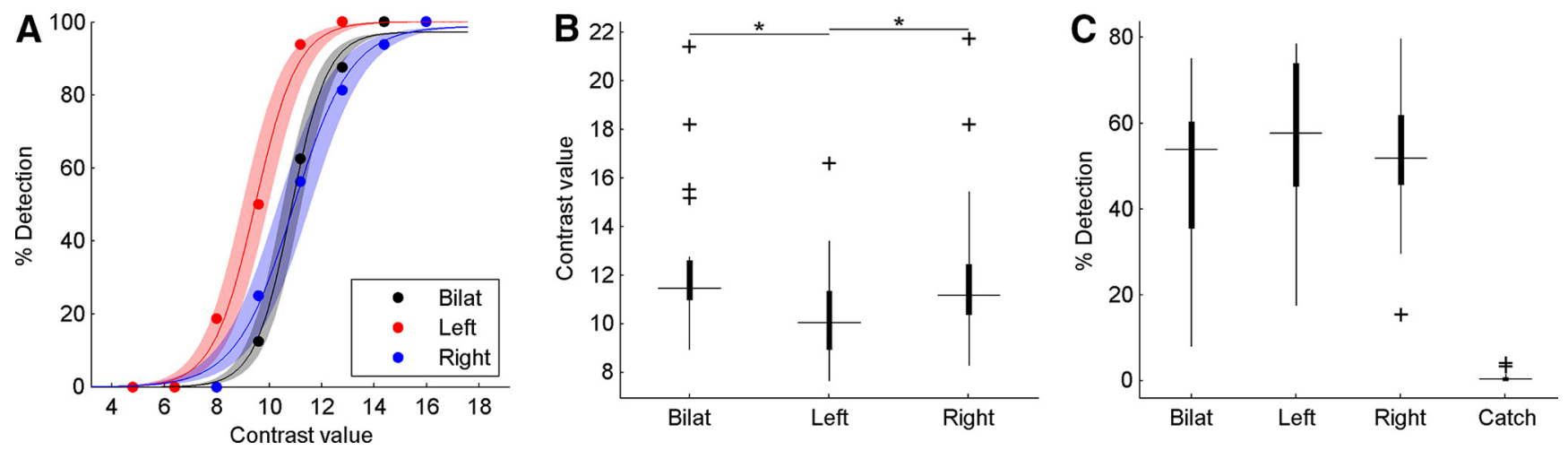

Figure 3. Behavioral results. Sensitivity curves for 1 subject $(\boldsymbol{A})$, threshold contrast values $(\boldsymbol{B})$, and detection rates $(\boldsymbol{C})$ for all subjects and conditions. $* p<0.05$.

For Experiment 2, an ROI combining M1 and the dorsal premotor area contralateral to the moved arm was used (Mayka et al., 2006). Analysis of FC was conducted as described previously (Guggisberg et al., $2011,2015)$. We used the absolute imaginary component of coherence as index of FC and calculated the weighted node degree (WND) for each voxel as the sum of its coherence with all other cortical voxel (Newman, 2004). FC values can be influenced by the signal-to-noise ratio of the EEG. To minimize this potential confound, we normalized WND values by calculating $z$ scores. This was achieved by subtracting the mean WND value of all voxels of the subject from the imaginary component of coherence values at each voxel and by dividing by the SD over all voxels (Dubovik et al., 2012; Mottaz et al., 2015).

The $\alpha$ frequency band $(8-12 \mathrm{~Hz})$ was the primary band of interest. Other frequency bands $(\theta, \beta, \gamma$, high- $\gamma$; respectively, 3-7, 13-30, 31-45, and $55-100 \mathrm{~Hz}$ ) were included to assess band specificity. The high- $\gamma$ band was only examined in Experiment 1, as the ICA preprocessing of Experiment 2 needed to be limited to $1-40 \mathrm{~Hz}$.

Experiment 1. To compute overall levels of spontaneous FC, 1500 artifact-free resting-state epochs were randomly selected for each participant.

To examine variations of FC in and their influence on subsequent performance, we calculated FC values for each block, based on the last $12 \mathrm{~s}$ of each resting period, for a median of 116 (range 85-120) artifactfree blocks.

Task-induced power modulations were reconstructed for the peristimulus period from $500 \mathrm{~ms}$ before to $750 \mathrm{~ms}$ after target onset using the same pass bands and inverse solutions as for FC. A $200 \mathrm{~ms}$ long sliding window with a $50 \mathrm{~ms}$ time step was used. Single-trial power at the visual ROIs was computed as the average root-mean square of source time series across ROI voxels for a median of 190 (range 141-224) artifact-free trials for each subject and condition. Average values across trials were computed after baseline subtraction for each participant. In addition, mean power differences between seen and missed trials were obtained from non-baseline-corrected values to avoid confounds arising from baseline differences. They were computed as area under curve of the whole peristimulus period.

Experiment 2. Spontaneous FC was obtained from 300 artifact-free epochs of $1 \mathrm{~s}$ recorded during rest.

Task-induced power modulations were reconstructed from $562.5 \mathrm{~ms}$ before to $62.5 \mathrm{~ms}$ after the sequence cue, with 125 -ms-long sliding windows and $62.5 \mathrm{~ms}$ steps using 1-40 $\mathrm{Hz}$ bandpass data. Average power across 100 artifact-free epochs was obtained for each participant. In addition, the area under the curve of power change was obtained for time windows with significant differences between high and low FC participants.

Statistical analyses

Power values were log-transformed to obtain normal distributions.

To determine whether variations of our electrophysiological measures influence perception of subjects in Experiment 1, one-sample $t$ tests were applied on the difference in FC and in event-related power between seen and missed trials of left and right stimulation conditions. Correction for multiple comparisons was achieved through a 5\% FDR.
For assessment of the influence of coupling states in single trials on perception we performed, for each subject, a logistic regression using single-trial data from left and right conditions with spontaneous $\alpha$ band FC and in-task $\alpha$ band power change from baseline as predictors, and perception (seen vs missed) as binary outcome. The regression coefficients of each subject were then fed to a second-level analysis using Wilcoxon signed rank tests against the null hypothesis of 0 median. In addition, single trials were dichotomized to high and low FC states depending on their $\alpha$ band FC in the current block, using the median value of each subject as cutoff. In our task blocks, two visual stimulation trials per condition followed one resting period. In cases where both trials were artifact-free, power changes were associated with the same FC value regardless of their behavioral outcome.

Between-subject Pearson correlations were used to test the relations between subject's mean values of performance, spontaneous $\alpha$ band FC traits, and the area under curve of in-task $\alpha$ band power decreases, for data from both experiments. No outliers were present that could have biased the correlation estimates. To further explore those relations, we split the subject sample in halves using the median resting-state $\alpha$ band FC level at the ROI as group cutoff criteria (low vs high FC trait). In Experiment 1, we examined the within factors perception (seen vs missed) and time on in-task power as outcome with repeated-measures ANOVAs, using SPSS version 25 (IBM). In Experiment 2, we compared event-related power in high versus low FC subjects using repeated-measures ANOVAs. ANOVAs are reported with corrected degrees of freedom (Greenhouse-Geisser correction) for sphericity violation when necessary.

\section{Resource availability}

NUTMEG, the MATLAB toolbox used for these analyses, is available at http://nutmeg.berkeley.edu.

\section{Results}

\section{Experiment 1}

The behavioral results for the visual detection task are illustrated in Figure 3. False alarm rates were very low in all participants (range $0 \%-4.2 \%$, median $=0.4 \%$ ) and Grier's B" values ranged from 0.711 to 1 (median $=0.97$ ), showing they all adopted a conservative response criteria as instructed. One-sample $t$ tests showed that performances in left, right, and bilateral stimulation conditions did not differ from $50 \%$ accuracy (all $p>0.11$ ), confirming that we were able to display perithreshold contrasts to our participants.

Task-induced activation and spontaneous network coupling We first confirm that visual target presentation induced, on average, the classical $\alpha$ band power decrease at bilateral visual areas after left as well as right target presentation compared with a baseline just before target presentation (Fig. $4 A, B$ ). When assessing all frequency bands at an anatomically defined, bilateral, occipital ROI (Fig. 4), one can observe the expected classical 
A

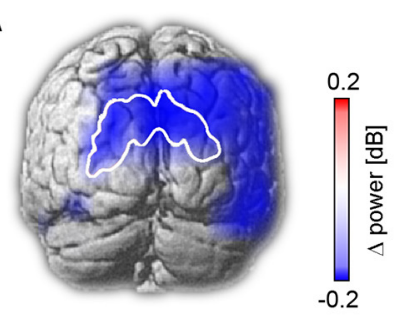

B

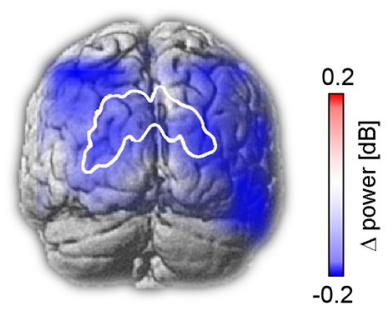

Frequency $[\mathrm{Hz}]$

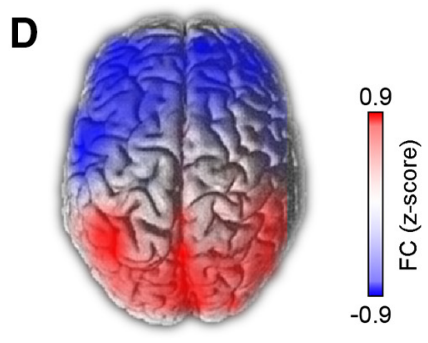

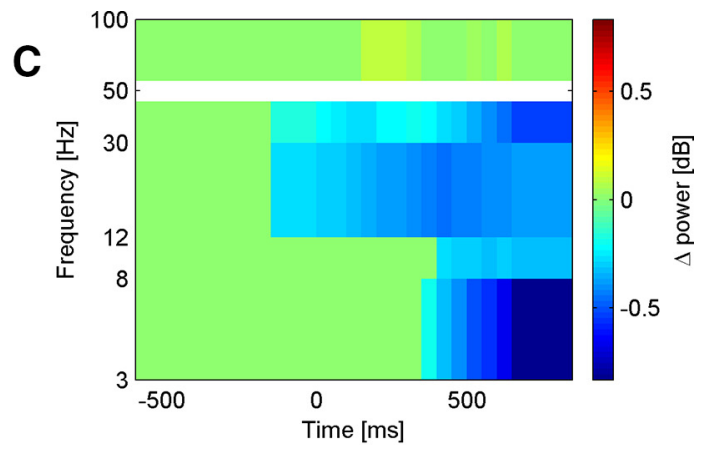

Time [s]

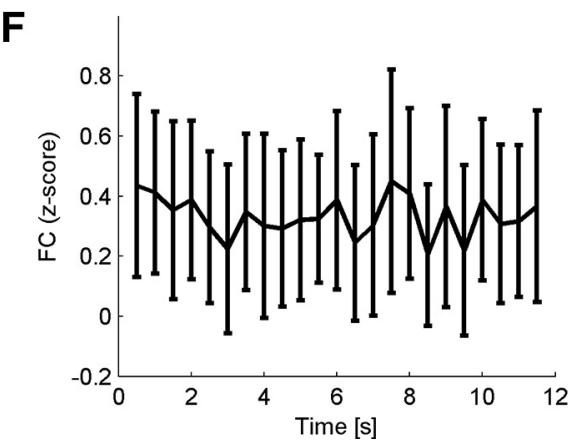

Figure 4. Characteristics of investigated neural processes. Average event-related in-task power changes induced by targets presented to the left $(\boldsymbol{A})$ and right $(\boldsymbol{B})$ with white overlaid ROI cutout. Blue represents regions with significant power decrease from baseline in the $\alpha$ band after the presentation of a target ( $p<0.05$, 5\% FDR-corrected over all voxels) on a 3D rendering of a template brain. Time-frequency decomposition of power changes in a bilateral occipital ROI compared with a baseline at 500 - $300 \mathrm{~ms}$ before target onset, as induced by a left or right visual stimulus ( $\boldsymbol{C} ; p<0.05,5 \%$ FDR-corrected over time and frequency). $\boldsymbol{D}$, The topography of resting-state $\alpha$ band FC shows an occipitofrontal gradient. $\boldsymbol{E}$, Frequency spectrum of resting-state FC in a bilateral occipital ROI. $\boldsymbol{F}, \mathrm{A}$ band $\mathrm{FC}$ variation over the interblock resting periods. Error bars indicate mean $\pm 95 \% \mathrm{Cl}$.

task-induced activation pattern, consisting of enhanced high- $\gamma$ activity and suppressed activity at slower bands (Fig. 4C). Since low-frequency power decreases for left and right targets were bilateral (see Discussion), we henceforth primarily report results based on pooled data from left and right conditions at the bilateral occipital ROI. Main analyses were however also performed for both conditions separately and were consistent after left and right target presentation.

When assessing global resting-state FC (i.e., the WND) of each brain area, we reproduced the occipitofrontal gradient (Fig. 4D) and predominance at $\alpha$ frequencies (Fig. $4 E$ ) described previously (Guggisberg et al., 2008; Hillebrand et al., 2012). A repeated-measures one-way ANOVA of WND at the ROI revealed a significant effect of frequency band $\left(F_{(4,76)}=33.34, p<0.001\right)$ with FC values significantly higher in the $\alpha$ band than in all other bands $(p<0.05$, Tukey-Kramer HSD). This confirms that the $\alpha$ band was the preferential frequency for neural communication (Chapeton et al., 2019). A repeated-measures one-way ANOVA showed no effect of time across the $12 \mathrm{~s}$ of interblock resting periods $\left(F_{(22,418)}=0.56\right.$, $p>0.9)$, hence demonstrating that the $\alpha$ band FC was stable during resting periods (Fig. $4 F$ ).

\section{ERD and FC states predict perception}

Seen trials induced on average greater event-related power decrease in $\alpha, \theta$, and $\beta$ bands in the poststimulus period than missed trials $(p<0.05$, corrected; Fig. $5 A$ ). Spontaneous FC was greater during resting periods before seen than before missed trials in the $\alpha$ frequency band $\left(t_{(19)}=3.03, p=0.035\right.$, corrected), but not in the other frequency bands (all uncorrected $p>0.05$ Fig. $5 B$ ). Importantly, resting-state $\alpha$ band power at the ROI did not differ before seen versus missed trials $(p=0.9)$.
A logistic regression of single trials with conscious perception as binary outcome, performed for each subject, confirmed that high $\alpha$ band FC states $(p=0.023)$ and $\alpha$ band power decrease from baseline $(p=0.021)$ at the ROI predicted better visual perception.

ERD and FC traits have opposite effects on visual performance We then subjected $\alpha$ band FC at rest and difference in task-induced $\alpha$ band power between seen and missed trials identified in the previous screening steps to between-subject covariations between behavioral and neural levels. This confirmed that subjects with greater resting-state FC in the $\alpha$ band at the bilateral occipital ROI had proportionally better visual perception percentage $\left(r_{(18)}=0.45\right.$, $p=0.045$; Fig. 6A). Greater resting-state FC in the $\alpha$ band was also linked to better visual perception percentage for left $\left(r_{(18)}=0.46\right.$, $p=0.042)$ and right $\left(r_{(18)}=0.45, p=0.047\right)$ targets taken separately. Importantly, this was not the case for local resting-state $\alpha$ power $\left(r_{(18)}=0.10, p=0.66\right)$, or FC at any nonoccipital ROI $\left(\left|r_{(18)}\right|<0.37\right.$, $p>0.10)$. Conversely, subjects with greater in-task power decrease in seen than missed trials in $\theta\left(r_{(18)}=0.45, p=0.045\right), \alpha\left(r_{(18)}=\right.$ $0.37, p=0.10)$, and $\beta$ bands $\left(r_{(18)}=0.47, p=0.038\right)$ tended to perform proportionally worse in visual perception percentage (Fig. $6 B-$ $D)$. Thus, classical local in-task activation was associated with worse task performance while abundant spontaneous interactions were present in subjects requiring particularly low contrasts for correct visual perception.

High spontaneous network coupling trait reduces the need for intask activation

To examine underlying reasons for the inverse impact of restingstate $\alpha$-FC and in-task power differences on visual performance, 

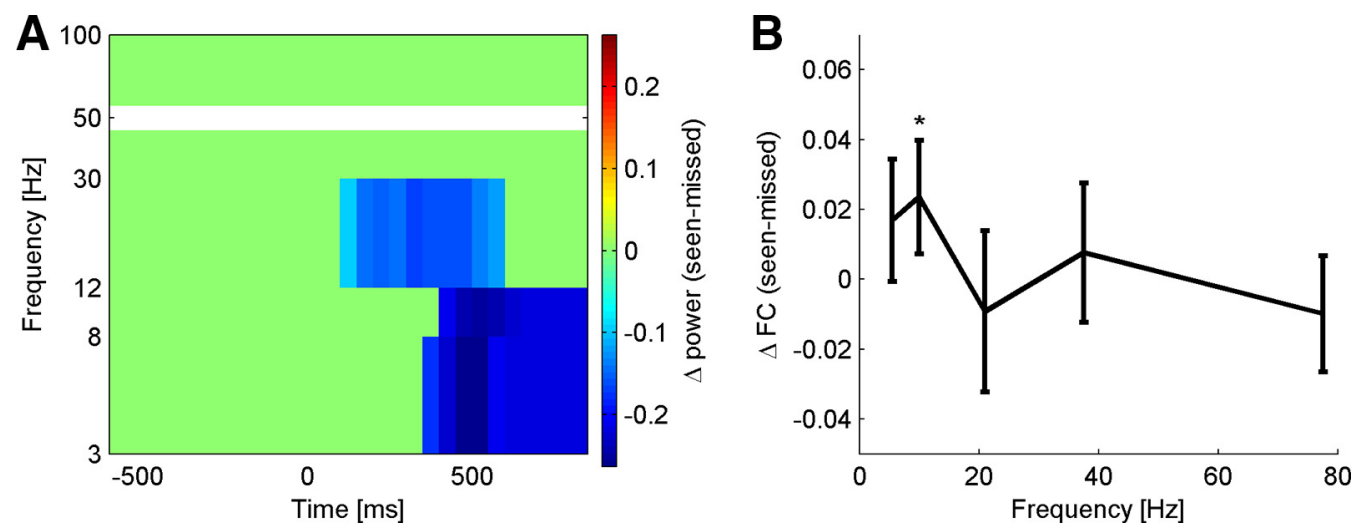

Figure 5. Neural processes predicting visual perception. Seen stimuli were associated, on average across all subjects, with greater power changes in $\theta, \alpha$, and $\beta$ bands during the presentation of seen stimuli than missed trials ( $\boldsymbol{A} ; p<0.05,5 \% \mathrm{FDR}$-corrected), in accordance with the classical pattern of task-induced neural activations. We also observed greater $\alpha$ band coherence between the ROI and the entire cortex during resting periods before perceived stimuli than before missed trials ( $\boldsymbol{B} ; * p=0.035,5 \%$ FDR-corrected), in accordance with previous findings of a positive impact of $\alpha$ band coherence during rest for task performance (Guggisberg et al., 2015). Error bars indicate mean $\pm 95 \% \mathrm{Cl}$.

we divided the participants in two equal groups based on their levels of resting-state $\alpha$-FC (median cutoff). This showed that only subjects with low $\alpha$ band FC demonstrate $\alpha$ power decrease after target presentation (Fig. 6E). In these subjects, a two-way repeated-measures ANOVA shows a main effect of time $\left(F_{(2.0,18.1)}=5.6, p=0.012\right)$ and perception $\left(F_{(1,9)}=26.2, p=0.001\right)$ and an interaction $\left(F_{(3.7,33.5)}=5.4, p=0.002\right)$, suggesting significant power decrease over time, which was greater during seen than during missed trials. Conversely, subjects with high spontaneous $\alpha$ band FC actually showed an absence of $\alpha$ power decrease following stimulus presentation, regardless of their awareness of the target (Fig. $6 F$ ). A two-way ANOVA shows no effect of time $\left(F_{(1.4,12.4)}=0.2, p=0.7\right)$ or perception $\left(F_{(1,9)}=0.4\right.$, $p=0.5)$, and no interaction $\left(F_{(4.1,36.6)}=1.0, p=0.4\right)$. The same conclusion had to be drawn for an explorative analysis of in-task power in all other frequency bands, as no frequency band showed significant differences between seen and missed trials in participants with high spontaneous $\alpha$ band FC (Fig. 7). Moreover, a voxelwise analysis of the whole brain did not find any area with significant $\alpha$ power decrease from baseline $(p>0.5$, corrected) in this subject group.

Bivariate correlation analyses between the two neural patterns further demonstrated that subjects with higher levels of $\alpha$-FC needed proportionally less power decrease in seen trials compared with missed trials in $\alpha\left(r_{(18)}=0.73, p=0.008\right.$, corrected; Fig. $6 G)$, as well as $\beta\left(r_{(18)}=0.69, p=0.009\right.$, corrected), and high $\gamma$ bands $\left(r_{(18)}=0.57, p=0.047\right.$, corrected). The association between $\alpha$-FC and $\alpha$ power was reproduced when analyzing left $\left(r_{(18)}=0.58, p=0.008\right)$ and right targets $\left(r_{(18)}=0.60, p=0.005\right)$ separately. An association was also found between $\gamma$ resting-state FC and power difference in $\alpha\left(r_{(18)}=0.63, p=0.026\right.$, corrected), and high- $\gamma\left(r_{(18)}=0.57, p=0.047\right.$, corrected). Crucially, unlike $\alpha$-FC, local $\alpha$ power during the resting periods ( $p>0.69$, corrected) or FC in the other frequency bands did not correlate with in-task power differences in any band (Fig. $6 H ; p>0.5$, corrected). Thus, the correlation was not merely because of a trivial dependency between FC and power or to mathematical coupling of a difference value.

\section{FC state versus trait effects}

Thus, both within-subject and between-subject analyses showed that neural $\alpha$ band coupling facilities conscious perception. This raises the question whether it is longer-lasting FC traits, present or absent in a given individual, or shorter-lasting FC states, fluctuating across time, that are important. Figure 8 illustrates that both FC states and FC traits define the need for local ERDs and influence perception, but in different ways. Subjects with high $\alpha$ band FC traits show good perception performance and low $\alpha$ band ERD amplitudes regardless of the momentary FC state. In these subjects, single-trial logistic regression coefficients of FC states $(p=0.11)$ and ERD amplitudes $(p=0.85)$ on visual perception were not different from 0 . Conversely, subjects with globally low $\alpha$ band traits can induce momentary states of higher $\alpha$ band coupling, which then enables larger ERD amplitudes $\left(F_{(3,36)}=\right.$ $4.7, p=0.0007$ ). In single-trial logistic regression analyses of these subjects, both FC states $(p=0.037)$ and ERD amplitude $(p=0.0039)$ predicted visual perception.

\section{Network correlates of leftward spatial attention bias}

We confirmed the presence of a leftward visuospatial bias in our paradigm. A Friedman test showed a significant effect of the condition on threshold contrast values $\left(\chi_{(2)}^{2}=27.70, p<0.001\right)$, as threshold values for left targets were lower than for right and bilateral targets $(p<0.05$, Tukey-Kramer HSD). We then assessed whether this behavioral bias was also reflected in spontaneous network interaction, both on the single-trial and the subject level.

As predicted, a negative correlation was found between resting-state $\alpha$-FC in the parietal ROI and the visuospatial leftward bias $\left(r_{(18)}=-0.49, p=0.027\right.$; Fig. $\left.9 B\right)$. Difference in $\alpha$ power during the task, in contrast, did not significantly correlate with the visuospatial leftward bias $\left(r_{(18)}=-0.33, p=0.15\right)$. Regarding unilateral targets, one-sample $t$ tests showed that the difference in $\mathrm{FC}$ in the ROI before seen versus missed trials was significant for left targets, but not for right targets (respectively, $p=0.025$ and $p=0.73$, corrected; Fig. $9 C$ ). The same analysis regarding power difference between $500 \mathrm{~ms}$ before to $750 \mathrm{~ms}$ after stimulus presentation showed no significant difference (left: $p=0.16$; right: $p=0.21$, corrected).

These findings demonstrate that participants with greater $\alpha$ band coupling in the right intraparietal sulcus overall showed less spatial bias toward the left visual field. On a trial-by-trial basis, this effect is apparent on unilateral targets, for which we show that greater coupling before stimulus presentation disfavors perception of left, but not right, targets. We suggest that greater coupling of the parietal ROI allows a stronger correction of the spontaneous leftward attentional bias when the task requires bilaterally distributed attention. At the trial level, variations of said coupling mediate the overall advantage shown for left targets. 

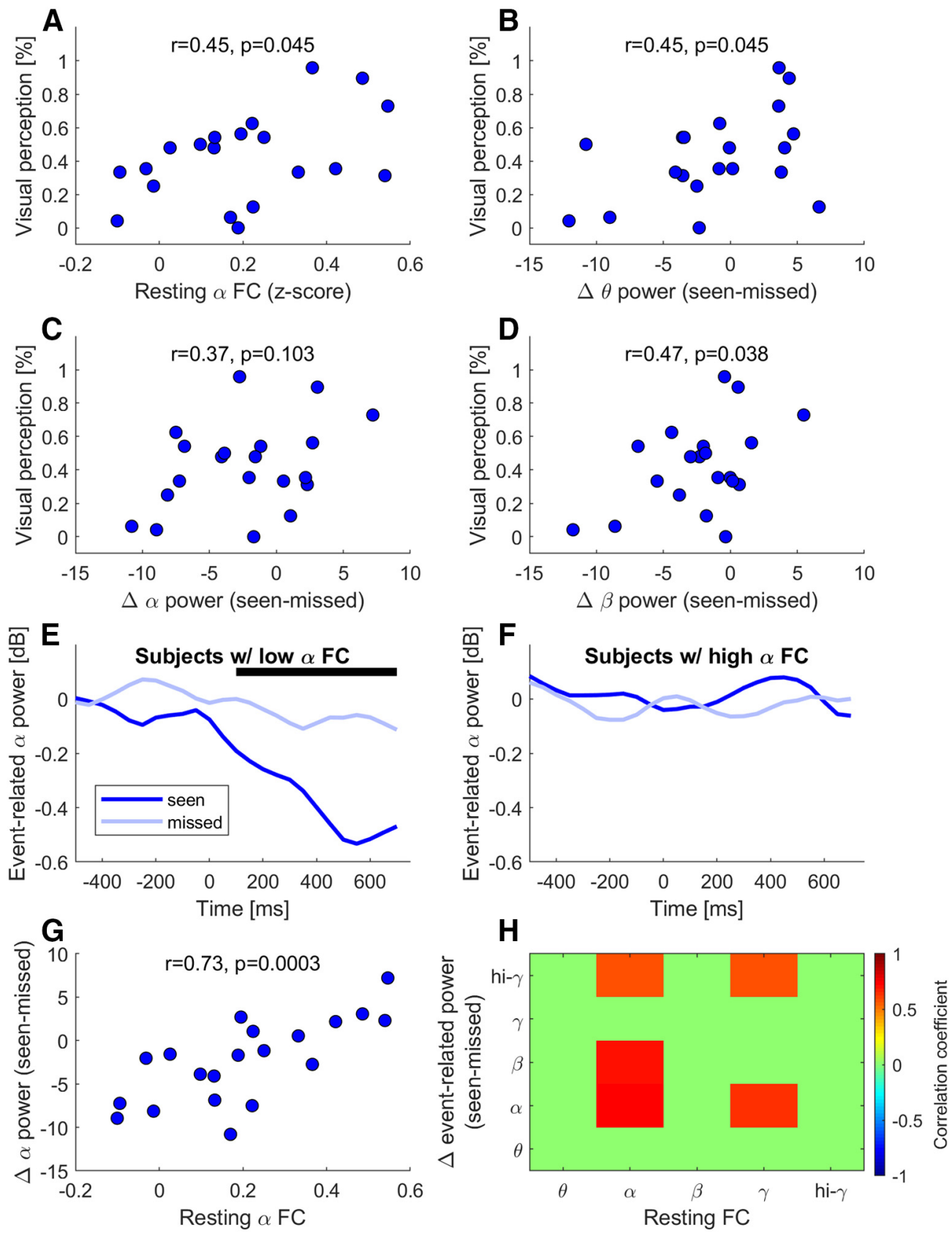

Figure 6. Between-subject covariation of neural processing and target perception. $\boldsymbol{A}$, Subjects with greater spontaneous $\alpha$ band WND in the occipital ROl showed proportionally better visual detection rates. $\boldsymbol{B}-\boldsymbol{D}$, Conversely, subjects with greater in-task $\theta, \alpha$, and $\beta$ power difference (seen - missed) tended to have proportionally lower detection rates. $\boldsymbol{E}$, Dividing the sample in two equal groups based on their levels of resting-state $\alpha$-FC (median cutoff) showed that only subjects with low $\alpha$ band $\mathrm{FC} \mathrm{level} \mathrm{demonstrate} \mathrm{the} \mathrm{classical} \mathrm{task-induced} \mathrm{power} \mathrm{decrease.} \mathrm{Black}$ line indicates time windows in which the difference between seen and missed trials is significant ( $p<0.05,5 \%$ FDR-corrected over time). $\boldsymbol{F}$, Conversely, subjects with high $\alpha$ band FC show an absence of $\alpha$ power decrease following stimulus presentation, regardless of their awareness of the target. Association between resting-state $\alpha$ band FC and in-task $\alpha$ power difference (G) and same association for all bands ( $\boldsymbol{H}$; Pearson correlations, $\boldsymbol{p}<0.05,5 \%$ FDR-corrected).

\section{Interindividual differences in FC}

Given the prominent impact of spontaneous network coupling, we were interested in factors that might promote it. An explorative analysis did not reveal significant effects of age, gender, hours of sleep the night preceding the recording, or subjective fatigue during the task ( $p>0.29$, corrected). The amount of preexisting experience with visual discrimination might contribute to spontaneous neural coupling but was not quantified in subjects of Experiment 1 .

\section{Experiment 2}

The second experiment allowed us to clarify this point. As 9 participants had previous experience with piano playing (median
12 years, range 1-12 years), we were able to test the influence of previous experience with motor sequence tasks on FC. Experiment 2 further intended to demonstrate that our findings obtained with visual perception generalize to other task types.

The finger tapping task induced, on average, robust ERDs in $\theta, \alpha, \beta$, and $\gamma$ bands during the motor planning phase in centroparietal brain areas contralateral to the moved hand (Fig. $10 A$ ) and in primary motor areas in particular (Fig. 10B). ERDs in motor ROIs did not correlate with performance in this task in any frequency band $\left(\left|r_{(18)}\right|<0.39, p>0.085\right)$. Conversely, when investigating spontaneous $\alpha$ band coupling as reconstructed from $5 \mathrm{~min}$ of resting-state recording, we reproduced for motor 


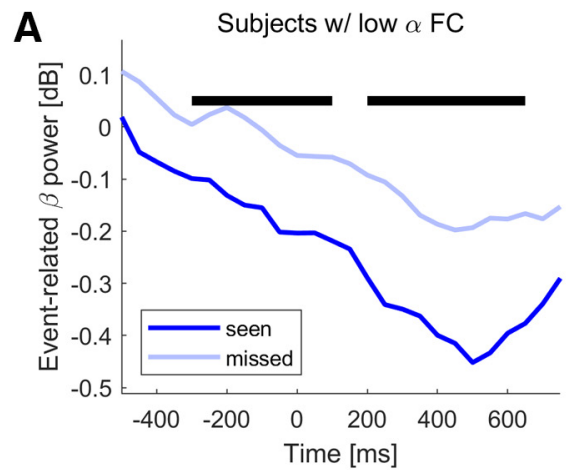

B
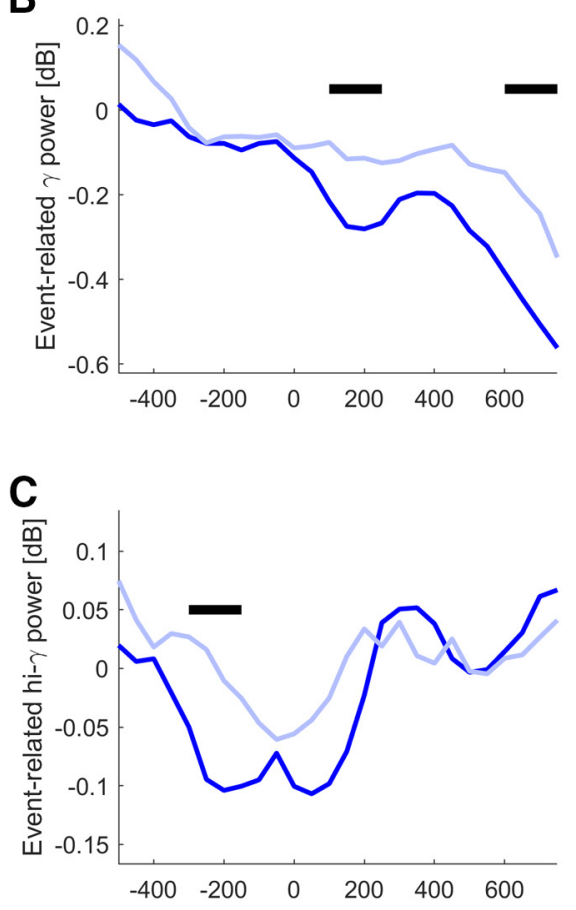
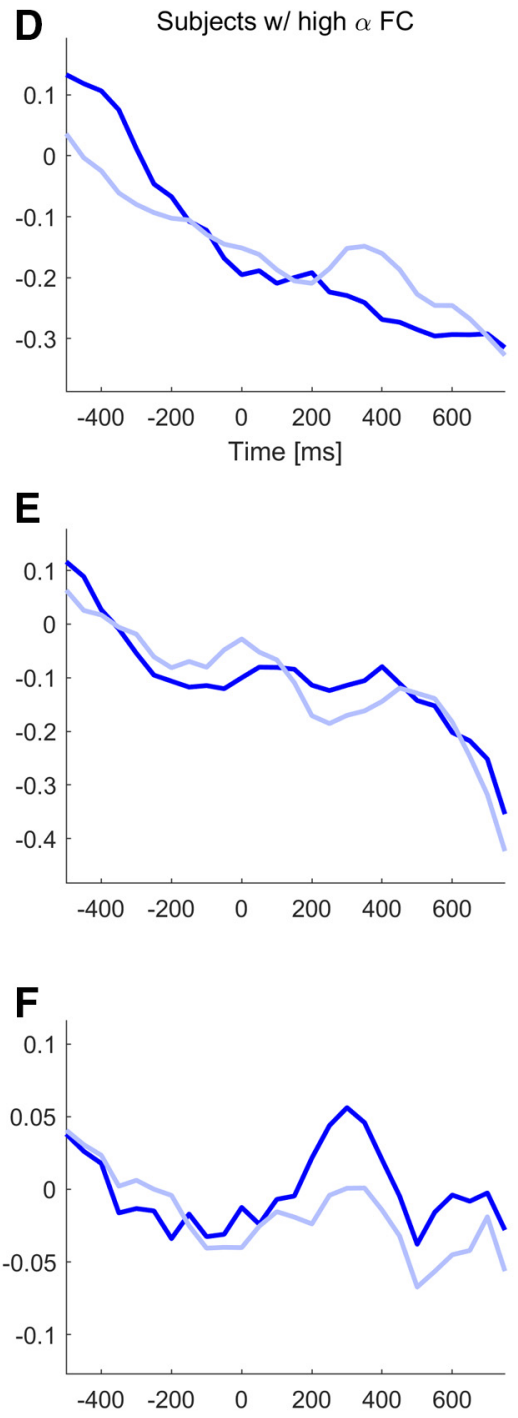

H
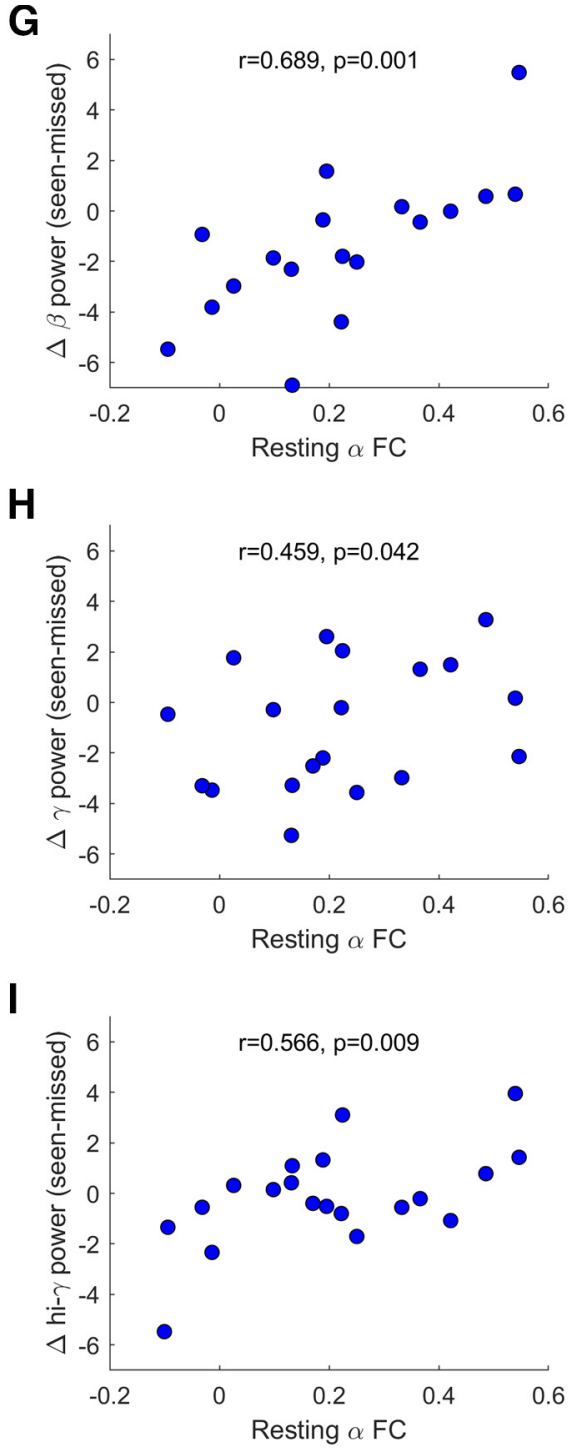

Figure 7. Generalization to task-induced power in other frequency bands. Power variation over the peristimulus interval for seen and missed targets in $\beta$, $\gamma$, and high- $\gamma$ in the 10 subjects with the lowest $(\boldsymbol{A}-\boldsymbol{C})$ or highest $(\boldsymbol{D}-\boldsymbol{F})$ resting-state $\alpha$ WND. Black line indicates time windows in which the difference between seen and missed trials is significant $(p<0.05)$. Association between resting-state $\alpha$ WND and in-task power difference in $\beta(\boldsymbol{G}), \gamma(\boldsymbol{H})$, and high- $\gamma$ frequency bands (I; Pearson correlations).

skills that spontaneous $\alpha$ band FC of the motor ROI with the rest of the brain correlated with performance (the average number of completed sequences per minute) of the participants (Fig. 10C; $r_{(18)}=0.48, p=0.033$ ). This was again not the case for FC in other frequency bands or in any nonmotor ROI of the automated anatomical labeling atlas $\left(\left|r_{(18)}\right|<0.38, p>0.1\right)$.

Crucially, $\alpha$ band FC, but not FC in the other frequency bands, correlated with the number of years of piano playing (Fig. $10 D)$, hence suggesting that previous experience with the task was linked to the appearance of abundant $\alpha$ band coupling. Piano experience was also positively correlated with performance $(\rho=0.77, p<0.0001)$.

When dividing the subjects into two equal subgroups of high and low spontaneous $\alpha$ band FC (median cutoff), we again observed that subjects with low spontaneous FC needed greater power decrease in $\theta\left(F_{(1,189)}=9.4, p=0.0025\right), \alpha\left(F_{(1,189)}=18.1\right.$, $p<0.0001)$, and $\beta$ bands $\left(F_{(1,189)}=12.2, p=0.0006\right)$ than participants with high FC (Fig. 10E). Furthermore, the amount of spontaneous $\alpha$ band FC correlated with the area under curve of ERDs in all examined frequency bands (Fig. 10F), similarly as in Experiment 1.

\section{Discussion}

We reproduced, on average, the classical local power decrease (i. e., ERD), which is widely used as an index of cortical activation (Pfurtscheller and Lopes da Silva, 1999). At the single-trial level, ERDs had a positive, independent impact on perception in a within-subject analysis. However, this result was driven by only part of the sample, as some subjects show little to no ERDs following target presentation, whether that target was seen or missed. Furthermore, if post-target ERD is indeed involved in perception or action planning in some subjects, our results show that it is not the most efficient processing pathway. Indeed, the subjects who were able to detect the highest percentage of targets of a given contrast, or to complete the greatest number of motor sequences, were those in which the event-related power decrease was the smallest, tending toward an absence of difference.

We also reproduce previous findings that higher network coupling enables efficient neural processing and high performance (Hipp et al., 2011; Weisz et al., 2014; Guggisberg et al., 2015; Sadaghiani et al., 2015; Strauß et al., 2015). However, previous research has not examined the relative importance of 

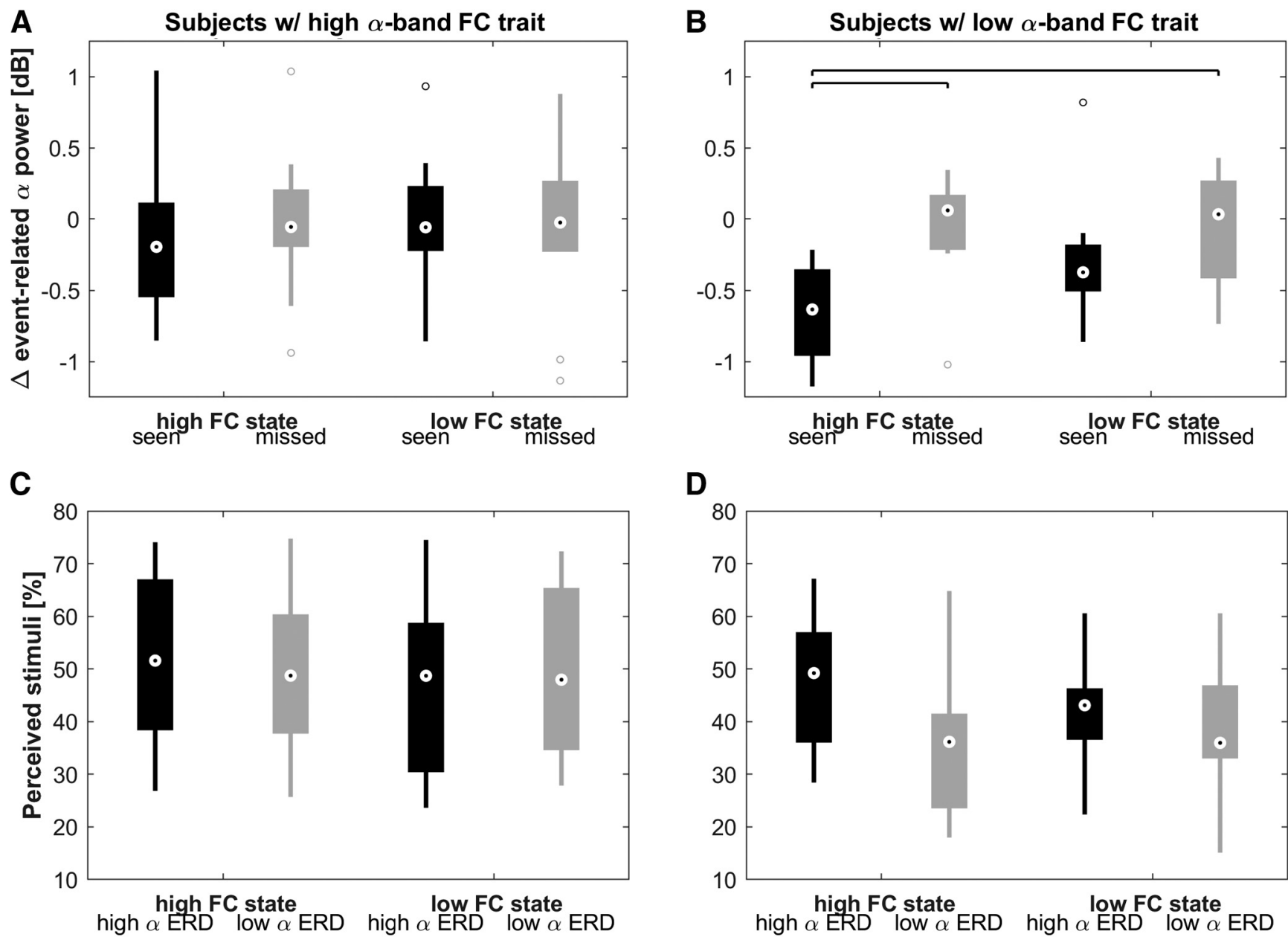

Figure 8. Comparison of the influence of $\alpha$ band FC traits and FC states on task-induced $\alpha$ band ERD and visual perception. Subjects with high $\alpha$ band FC traits show low $\alpha$ band ERD amplitudes and stable perception performance regardless of the momentary FC state $(\boldsymbol{A}, \boldsymbol{C})$. Subjects with low FC traits had blocks with higher $\alpha$ band FC states, which were associated with significantly greater $\alpha$ band ERDs $(\boldsymbol{B})$. Horizontal lines indicate significant differences ( $p<0.05$, Tukey-Kramer HSD) and, on average, a nonsignificantly larger proportion of perceived stimuli (D).

\section{A}

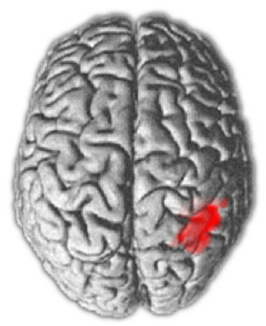

B

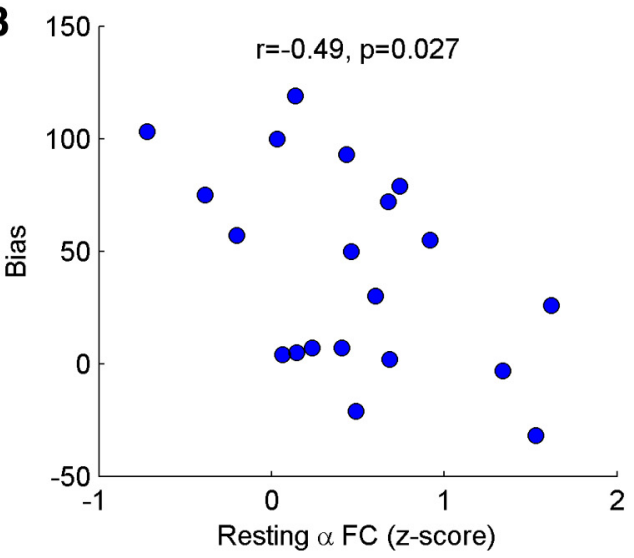

C

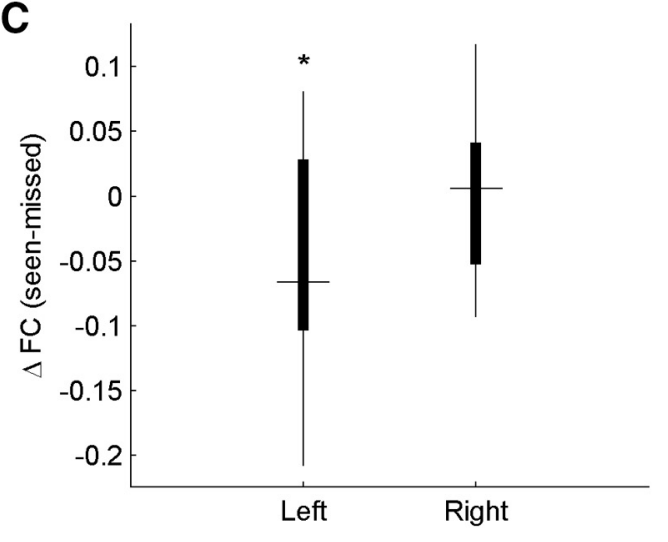

Figure 9. Spontaneous network correlates of leftward spatial attention bias. $\boldsymbol{A}$, Parietal ROI. $\boldsymbol{B}$, Pearson correlation between resting-state $\alpha$-WND of the parietal R0I and visuospatial leftward bias in bilateral stimulus presentations. C, Difference in $\alpha$-WND preceding unilateral stimulus presentations. $* p<0.05$ (5\% FDR-corrected).

connectivity compared with classical in-task activations. Moreover, FC states were induced externally by repetitive tasks.

Our article compares the impact of spontaneous FC across much longer time scales to the impact of classical task-induced brain activations on behavior. The critical novelty is that spontaneous FC, in particular $\alpha$ band phase coupling, are the primary correlate of task success enabling efficient distributed processing. This was the case for fluctuations of FC over several tens of seconds, during which states with higher FC were associated with better abilities to induce ERDs and to perceive the targets. And the effect was even more prominent in subjects with long-lasting traits of high coupling, in which case local ERDs became 
A
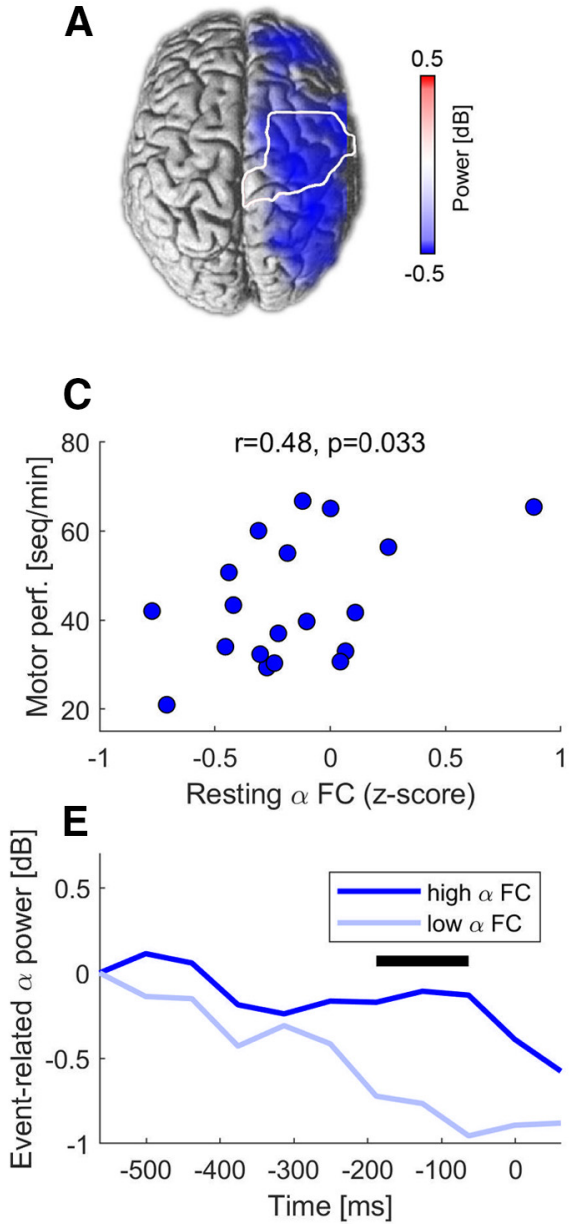
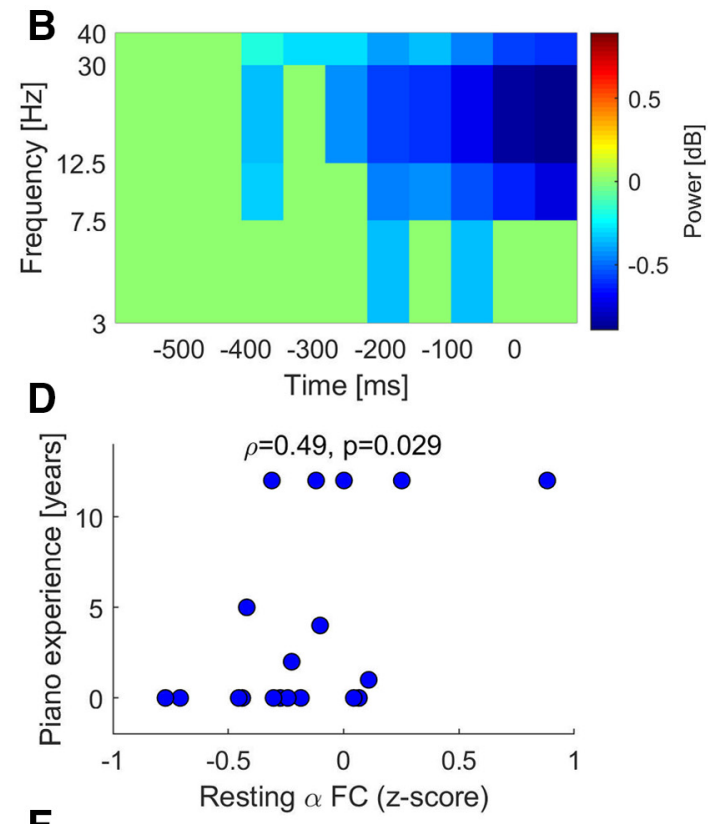

$\mathbf{F}$

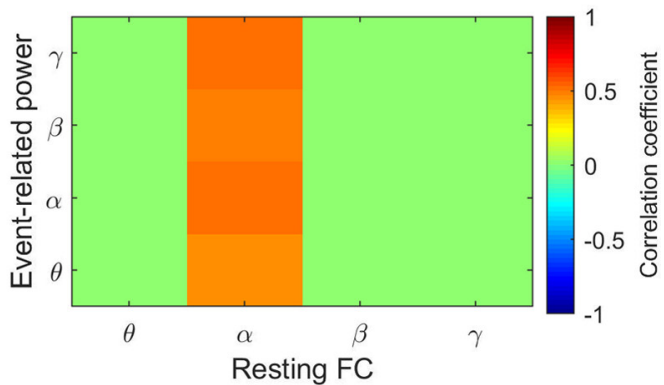

Figure 10. Generalization to motor planning. $\boldsymbol{A}$, Mean event-related $\alpha$ band power decrease before execution of a motor sequence across all 20 patients $(p<0.05,5 \%$ FDR-corrected over voxels) with white overlaid ROl cutout. $B, A$ time-frequency decomposition at the contralateral motor ROl shows, on average, prominent power decrease in all examined bands ( $p<0.05,5 \%$ FDR-corrected over time-frequency windows). Time 0 indicates the onset cue. C, Correlation between spontaneous $\alpha$ band FC and number of correct motor sequences per minute. $\boldsymbol{D}$, Greater $\alpha$ band $\mathrm{FC}$ was in turn associated with more years of experience with piano playing. $\boldsymbol{E}$, Participants with low spontaneous $\alpha$ band $\mathrm{FC}$ needed significantly greater event-related $\alpha$ power decrease than subjects with high FC. Black line indicates time points with significant difference (5\% FDR-corrected). Thus, greater spontaneous $\alpha$ band FC correlated with proportionally smaller taskinduced power decrease at all examined frequency bands ( $\boldsymbol{F} ; p<0.05$, corrected).

unnecessary and excellent behavioral performance became possible in both visual perception and motor tasks. This may reflect the underlying synaptic architecture with pre-established pathways for distributed processing of the task (Weisz et al., 2014). This also means that network traits and states need to be taken into account in any attempt to explain interindividual differences in behavior and task-related neural events. In contrast, activations induced by classical task contexts are minimal or absent in high performers, which demonstrates that they are merely a compensation mechanism. Thus, ERDs can be seen as index of effortful attention (Wyart and Tallon-Baudry, 2008), which enables low-performing subjects to accomplish a task despite unfavorable neural interactions. In the context of motor planning, ERDs could reflect hemispheric dominance or proprioception (Bai et al., 2005; Nakayashiki et al., 2014) rather than the actual motor command.

Evidence for a causal role of spontaneous coupling on performance comes from neurofeedback studies demonstrating that enhancing neural coupling improves behavioral performance in healthy (Kajal et al., 2017; Koush et al., 2017) and in patients with brain lesions (Mottaz et al., 2018). Thus, neural coupling may become a primary target in programs that aim to improve performance. Indeed, Experiment 2 shows that long-term experience with a task associates with traits of high spontaneous neural coupling of the involved brain areas. Future work will need to examine which training strategies are most efficient for enhancing spontaneous neural coupling. In particular, it may be possible to use complementary and more efficient methods to classical repetitive task training.

ERDs found after unilateral stimulation in Experiment 1 were bilateral. Conversely, activity induced by unilateral targets is classically shown to be contralateral to their presentation. However, several aspects of our paradigm may explain this. First, we provided no cueing on the location of the next target. The best strategy was thus to attend to both locations for all trials. Second, the level of validity of the cue (i.e., yielding a given spatial certainty) influences the degree of $\alpha$ power lateralization (Rohenkohl and Nobre, 2011). Since targets could be presented bilaterally in our task, the effect on $\alpha$ power is less lateralized than in paradigms that include only one-sided stimulations.

High- $\gamma$ power increase is, as well as $\alpha$ ERD, predictive of perceptual decisions (Wyart and Tallon-Baudry, 2009) and motor planning (Gunduz et al., 2016). Furthermore, high- $\gamma$ activations are usually more circumscribed and unilateral and more taskspecific (Crone et al., 2006). Figures $6 \mathrm{H}$ and 7 demonstrate that our conclusions also apply to high- $\gamma$ activations. 
Another classical electrophysiological measure of task-related activations are ERPs. Typically, ERP amplitudes are found to be greater for seen compared with missed trials in many paradigms (Railo et al., 2011). Amplitudes of early ERP components are furthermore related to the state of ongoing activity at stimulus onset (Ergenoglu et al., 2004; Iemi et al., 2019). How overall performance relates to ERPs is however still unclear. Conflicting results arise from different paradigms, and the influence also seems to be age-related (Hanslmayr et al., 2005; Riis et al., 2008; Wolk et al., 2009). While ERP analyses were not the focus of this work, it would be interesting to determine in future studies whether ERP amplitudes also depend on spontaneous network coupling.

New models of brain functioning are required to integrate our observations. The importance of network interactions for conscious processing, in particular for visual consciousness, is already well grounded in connectivist models, such as the global neuronal workspace theory of consciousness (Dehaene and Changeux, 2011) or the "Windows to Consciousness" framework (Ruhnau et al., 2014; Weisz et al., 2014). Notably, Rassi et al. (2019) recently showed that prestimulus $\alpha$ band connectivity biased the perception of an ambiguous stimulus, whereas prestimulus power did not, providing yet another example of the preponderant role of FC in defining ongoing states. However, our findings additionally demonstrate the importance of spontaneous FC outside of the task context.

How might spontaneous network FC influence behavior and determine the need for task-induced activations? The neural efficiency theory (Haier et al., 1988) suggests that higher intelligence is underpinned by a higher efficiency in its functioning rather than a higher excitability or $\alpha$ band ERD as an index of cortical activation (Grabner et al., 2004). This framework has been extended beyond the notion of intelligence to expertise: skilled populations (e.g., expert chess players or athletes) have a reduction in task-induced brain activation compared with naive subjects (Milton et al., 2007; Del Percio et al., 2010), in accordance with our findings from Experiment 2. Recent research identified neuronal coupling as underlying electrophysiological correlate of neural efficiency and expertise. Network interactions were found to be stronger in expert brains at rest (Gong et al., 2019) and during tasks related to their field of expertise (Bhattacharya and Petsche, 2005). In this view, $\alpha$ band coupling thus reflects general intelligence and expertise.

The predictive processing theory goes a step further in suggesting that expertise arises from a refined model of the self and world, which allows to make more accurate predictions about upcoming events (Friston and Stephan, 2007; Euler, 2018). In this framework, network interaction could be seen as reflecting expectancies (Mayer et al., 2016), which help reduce the indeterminacy of visual stimuli or motor sequences. This could in turn result in reduced ERDs reflecting less prediction error (i.e., less difference between expected and observed events). Although we did not modulate indeterminacy in our paradigms, it has been argued that past experience, as in our musicians of Experiment 2, will make low-contrast visual stimuli or motor sequences less novel and indeterminate (Euler, 2018).

It is interesting to note that the brain has a preference for $\alpha$ frequencies for long-range neural interactions. We underline that it is spontaneous $\alpha$ band coupling that predicted performance, whereas local $\alpha$ power did not. Therefore, the frequency specificity cannot be due to a higher signal-to-noise ratio of $\alpha$ activity. Rather, $\alpha$ seems to have a primary role in neural communication during rest, which we show here to enable high task performance. Most previous propositions on the role of $\alpha$ activity have been derived from classical task-related experiments, which is precisely the situation in which $\alpha$ is replaced by faster rhythms. The idea of an inhibitory role may thus be an artifact resulting from the experimental context (Palva and Palva, 2011).

In addition to the $\alpha$-frequency that was of primary interest here, we also observe that phase coupling in $\gamma$ frequencies influences ERD in visual areas (Fig. 5H). This matches well with animal studies: intracortical recordings in monkey visual cortex have shown that $\alpha$ exerts a top-down modulation on visual processing, through its influence on $\gamma$ activity. Within V1, these two rhythms display phase-amplitude coupling as well as anticorrelated power (Spaak et al., 2012). $\gamma$ oscillations travel downstream from primary (V1) to secondary (V4) areas, whereas $\alpha$ oscillations flow upstream (van Kerkoerle et al., 2014). In relation with our results, we can thus hypothesize that a high sensitivity to feedback from higher level to primary areas, as evidenced by a high level of FC in the $\alpha$ band, is an advantage for stimulus detection.

\section{References}

Babiloni C, Vecchio F, Bultrini A, Romani GL, Rossini PM (2006) Pre- and poststimulus alpha rhythms are related to conscious visual perception: a high-resolution EEG study. Cereb Cortex 16:1690-1700.

Bai O, Mari Z, Vorbach S, Hallett M (2005) Asymmetric spatiotemporal patterns of event-related desynchronization preceding voluntary sequential finger movements: a high-resolution EEG study. Clin Neurophysiol 116:1213-1221.

Bhattacharya J, Petsche H (2005) Phase synchrony analysis of EEG during music perception reveals changes in functional connectivity due to musical expertise. Signal Processing 85:2161-2177.

Brainard DH (1997) The psychophysics toolbox. Spat Vis 10:433-436.

Chapeton JI, Haque R, Wittig JH, Inati SK, Zaghloul KA (2019) Large-scale communication in the human brain is rhythmically modulated through alpha coherence. Curr Biol 29:2801-2811.e5.

Choi HJ, Zilles K, Mohlberg H, Schleicher A, Fink GR, Armstrong E, Amunts K (2006) Cytoarchitectonic identification and probabilistic mapping of two distinct areas within the anterior ventral bank of the human intraparietal sulcus. J Comp Neurol 495:53-69.

Crone NE, Sinai A, Korzeniewska A (2006) High-frequency gamma oscillations and human brain mapping with electrocorticography. Prog Brain Res 159:275-295.

Dalal SS, Zumer JM, Guggisberg AG, Trumpis M, Wong DD, Sekihara K, Nagarajan SS (2011) MEG/EEG source reconstruction, statistical evaluation, and visualization with NUTMEG. Comput Intell Neurosci 2011:758973.

Dehaene S, Changeux JP (2011) Experimental and theoretical approaches to conscious processing. Neuron 70:200-227.

Del Percio C, Infarinato F, Iacoboni M, Marzano N, Soricelli A, Aschieri P, Eusebi F, Babiloni C (2010) Movement-related desynchronization of alpha rhythms is lower in athletes than non-athletes: a high-resolution EEG study. Clin Neurophysiol 121:482-491.

Dubovik S, Pignat JM, Ptak R, Aboulafia T, Allet L, Gillabert N, Magnin C, Albert F, Momjian-Mayor I, Nahum L, Lascano AM, Michel CM, Schnider A, Guggisberg AG (2012) The behavioral significance of coherent resting-state oscillations after stroke. Neuroimage 61:249-257.

Dubovik S, Bouzerda-Wahlen A, Nahum L, Gold G, Schnider A, Guggisberg AG (2013) Adaptive reorganization of cortical networks in Alzheimer's disease. Clin Neurophysiol 124:35-43.

Eickhoff SB, Stephan KE, Mohlberg H, Grefkes C, Fink GR, Amunts K, Zilles K (2005) A new SPM toolbox for combining probabilistic cytoarchitectonic maps and functional imaging data. Neuroimage 25:1325-1335.

Ergenoglu T, Demiralp T, Bayraktaroglu Z, Ergen M, Beydagi H, Uresin Y (2004) Alpha rhythm of the EEG modulates visual detection performance in humans. Cogn Brain Res 20:376-383.

Euler MJ (2018) Intelligence and uncertainty: implications of hierarchical predictive processing for the neuroscience of cognitive ability. Neurosci Biobehav Rev 94:93-112.

Friston KJ, Stephan KE (2007) Free-energy and the brain. Synthese 159:417458. 
Gegenfurtner KR, Kiper DC, Levitt JB (1997) Functional properties of neurons in macaque area V3. J Neurophysiol 77:1906-1923.

Gillebert CR, Mantini D, Thijs V, Sunaert S, Dupont P, Vandenberghe R (2011) Lesion evidence for the critical role of the intraparietal sulcus in spatial attention. Brain 134:1694-1709.

Gong A, Liu J, Lua L, Wu G, Jiang C, Fu Y (2019) Characteristic differences between the brain networks of high-level shooting athletes and non-athletes calculated using the phase-locking value algorithm. Biomed Signal Process Control. 51:128-137.

Grabner R, Fink A, Stipacek A, Neuper C, Neubauer A (2004) Intelligence and working memory systems: evidence of neural efficiency in alpha band ERD. Brain Res Cogn Brain Res 20:212-225.

Greicius MD, Supekar K, Menon V, Dougherty RF (2009) Resting-state functional connectivity reflects structural connectivity in the default mode network. Cereb Cortex 19:72-78.

Grier JB (1971) Nonparametric indexes for sensitivity and bias: computing formulas. Psychol Bull 75:424-429.

Guggisberg AG, Honma SM, Findlay AM, Dalal SS, Kirsch HE, Berger MS, Nagarajan SS (2008) Mapping functional connectivity in patients with brain lesions. Ann Neurol 63:193-203.

Guggisberg AG, Dalal SS, Zumer JM, Wong DD, Dubovik S, Michel CM, Schnider A (2011) Localization of cortico-peripheral coherence with electroencephalography. Neuroimage 57:1348-1357.

Guggisberg AG, Rizk S, Ptak R, Di Pietro M, Saj A, Lazeyras F, Lovblad KO, Schnider A, Pignat JM (2015) Two intrinsic coupling types for resting-state integration in the human brain. Brain Topogr 28:318-329.

Gunduz A, Brunner P, Sharma M, Leuthardt EC, Ritaccio AL, Pesaran B, Schalk G (2016) Differential roles of high gamma and local motor potentials for movement preparation and execution. Brain-Computer Interfaces 3:88-102.

Haier RJ, Siegel BV, Nuechterlein KH, Hazlett E, Wu JC, Paek J, Browning HL, Buchsbaum MS (1988) Cortical glucose metabolic rate correlates of abstract reasoning and attention studied with positron emission tomography. Intelligence 12:199-217.

Hanslmayr S, Klimesch W, Sauseng P, Gruber W, Doppelmayr M, Freunberger R, Pecherstorfer T (2005) Visual discrimination perform ance is related to decreased alpha amplitude but increased phase locking. Neurosci Lett 375:64-68.

Hanslmayr S, Aslan A, Staudigl T, Klimesch W, Herrmann CS, Bäuml KH (2007) Prestimulus oscillations predict visual perception performance between and within subjects. Neuroimage 37:1465-1473.

Hillebrand A, Barnes GR, Bosboom JL, Berendse HW, Stam CJ (2012) Frequency-dependent functional connectivity within resting-state net works: an atlas-based MEG beamformer solution. Neuroimage 59:3909_ 3921.

Hipp JF, Engel AK, Siegel M (2011) Oscillatory synchronization in large-scale cortical networks predicts perception. Neuron 69:387-396.

Hyvarinen A (1999) Fast and robust fixed-point algorithms for independent component analysis. IEEE Trans Neural Netw 10:626-634

Iemi L, Busch NA, Laudini A, Haegens S, Samaha J, Villringer A, Nikulin VV (2019) Multiple mechanisms link prestimulus neural oscillations to sensory responses. Elife 8:e43620.

Jensen O, Mazaheri A (2010) Shaping functional architecture by oscillatory alpha activity: gating by inhibition. Front Hum Neurosci 4:186.

Kajal DS, Braun C, Mellinger J, Sacchet MD, Ruiz S, Fetz E, Birbaumer N, Sitaram R (2017) Learned control of inter-hemispheric connectivity: effects on bimanual motor performance. Hum Brain Mapp 38:43534369.

Klimesch W, Sauseng P, Hanslmayr S (2007) EEG alpha oscillations: the inhibition-timing hypothesis. Brain Res Rev 53:63-88.

Koush Y, Meskaldji DE, Pichon S, Rey G, Rieger SW, Linden DE, Van De Ville D, Vuilleumier P, Scharnowski F (2017) Learning control over emotion networks through connectivity-based neurofeedback. Cereb Cortex 27:1193-1202.

Kujovic M, Zilles K, Malikovic A, Schleicher A, Mohlberg H, Rottschy C, Eickhoff SB, Amunts K (2013) Cytoarchitectonic mapping of the human dorsal extrastriate cortex. Brain Struct Funct 218:157-172.

Larsson J, Heeger DJ (2006) Two retinotopic visual areas in human lateral occipital cortex. J Neurosci 26:13128-13142.

Learmonth G, Thut G, Benwell CS, Harvey M (2015) The implications of state-dependent tDCS effects in aging: behavioural response is determined by baseline performance. Neuropsychologia 74: 108-119.

Mayer A, Schwiedrzik CM, Wibral M, Singer W, Melloni L (2016) Expecting to see a letter: alpha oscillations as carriers of top-down sensory predictions. Cereb Cortex 26:3146-3160.

Mayka MA, Corcos DM, Leurgans SE, Vaillancourt DE (2006) Three-dimensional locations and boundaries of motor and premotor cortices as defined by functional brain imaging: a meta-analysis. Neuroimage 31:1453-1474

Milton J, Solodkin A, Hluštík P, Small SL (2007) The mind of expert motor performance is cool and focused. Neuroimage 35:804-813.

Mottaz A, Solcà M, Magnin C, Corbet T, Schnider A, Guggisberg AG (2015) Neurofeedback training of alpha band coherence enhances motor performance. Clin Neurophysiol 126:1754-1760.

Mottaz A, Corbet T, Doganci N, Magnin C, Nicolo P, Schnider A, Guggisberg AG (2018) Modulating functional connectivity after stroke with neurofeedback: effect on motor deficits in a controlled cross-over study. Neuroimage Clin 20:336-346.

Nakayashiki K, Saeki M, Takata Y, Hayashi Y, Kondo T (2014) Modulation of event-related desynchronization during kinematic and kinetic hand movements. J Neuroeng Rehabil 11:1-9.

Newman ME (2004) Analysis of weighted networks. Phys Rev E Stat Phys 70:056131.

Palva S, Palva JM (2011) Functional roles of alpha band phase synchronization in local and large-scale cortical networks. Front Psychol 2:204.

Pfurtscheller G, Lopes da Silva FH (1999) Event-related EEG/MEG synchronization and desynchronization: basic principles. Clin Neurophysiol 110:1842-1857.

Pfurtscheller G, Stancák A, Neuper C (1996) Event-related synchronization (ERS) in the alpha band-an electrophysiological correlate of cortical idling: a review. Int J Psychophysiol 24:39-46.

Railo H, Koivisto M, Revonsuo A (2011) Tracking the processes behind conscious perception: a review of event-related potential correlates of visual consciousness. Conscious Cogn 20:972-983.

Rassi E, Wutz A, Müller-Voggel N, Weisz N (2019) Prestimulus feedback connectivity biases the content of visual experiences. Proc Natl Acad Sci USA 116:16056-16061.

Riis JL, Chong H, Ryan KK, Wolk DA, Rentz DM, Holcomb PJ, Daffner KR (2008) Compensatory neural activity distinguishes different patterns of normal cognitive aging. Neuroimage 39:441-454.

Rohenkohl G, Nobre AC (2011) Alpha oscillations related to anticipatory attention follow temporal expectations. J Neurosci 31:1407614084.

Romei V, Gross J, Thut G (2010) On the role of prestimulus alpha rhythms over occipito-parietal areas in visual input regulation: correlation or causation? J Neurosci 30:8692-8697.

Ruhnau P, Hauswald A, Weisz N (2014) Investigating ongoing brain oscillations and their influence on conscious perception: network states and the window to consciousness. Front Psychol 5:1230.

Sadaghiani S, Poline JB, Kleinschmidt A, D’Esposito M (2015) Ongoing dynamics in large-scale functional connectivity predict perception. Proc Natl Acad Sci USA 112:8463-8468.

Scheperjans F, Eickhoff SB, Hömke L, Mohlberg H, Hermann K, Amunts K, Zilles K (2008) Probabilistic maps, morphometry, and variability of cytoarchitectonic areas in the human superior parietal cortex. Cereb Cortex 18:2141-2157.

Sekihara K, Nagarajan SS, Poeppel D, Marantz A (2004) Asymptotic SNR of scalar and vector minimum-variance beanformers for neuromagnetic source reconstruction. IEEE Trans Biomed Eng 51:1726-1734.

Spaak E, Bonnefond M, Maier A, Leopold DA, Jensen O (2012) Layer-specific entrainment of gamma band neural activity by the alpha rhythm in monkey visual cortex. Curr Biol 22:2313-2318.

Stanislaw H, Todorov N (1999) Calculation of signal detection theory measures. Behav Res Methods Instrum Comput 31:137-149.

Stenroos M, Mantynen V, Nenonen J (2007) A Matlab library for solving quasi-static volume conduction problems using the boundary element method. Comput Methods Programs Biomed 88:256-263.

Strauß A, Henry MJ, Scharinger M, Obleser J (2015) Alpha phase determines successful lexical decision in noise. J Neurosci 35:3256-3262. 
Thut G, Nietzel A, Brandt SA, Pascual-Leone A (2006) Alpha-band electroencephalographic activity over occipital cortex indexes visuospatial attention bias and predicts visual target detection. J Neurosci 26:9494-9502.

Treutwein B (1995) Adaptive psychophysical procedures. Vision Res 35:2503-2522.

van Dijk H, Schoffelen JM, Oostenveld R, Jensen O (2008) Prestimulus oscillatory activity in the alpha band predicts visual discrimination ability. J Neurosci 28:1816-1823.

van Kerkoerle T, Self MW, Dagnino B, Gariel-Mathis MA, Poort J, van der Togt C, Roelfsema PR (2014) Alpha and gamma oscillations characterize feedback and feedforward processing in monkey visual cortex. Proc Natl Acad Sci USA 111:14332-14341.
Weisz N, Wühle A, Monittola G, Demarchi G, Frey J, Popov T (2014) Prestimulus oscillatory power and connectivity patterns predispose conscious somatosensory perception. Proc Natl Acad Sci USA 111:E417E425.

Wolk DA, Sen NM, Chong H, Riis JL, McGinnis SM, Holcomb PJ, Daffner KR (2009) ERP correlates of item recognition memory: effects of age and performance. Brain Res 1250:218-231.

Wyart V, Tallon-Baudry C (2008) Neural dissociation between visual awareness and spatial attention. J Neurosci 28:2667-2679.

Wyart V, Tallon-Baudry C (2009) How ongoing fluctuations in human visual cortex predict perceptual awareness: baseline shift versus decision bias. J Neurosci 29:8715-8725. 